\title{
Secondary sclerosing cholangitis after chemotherapy with bevacizumab and paclitaxel
}

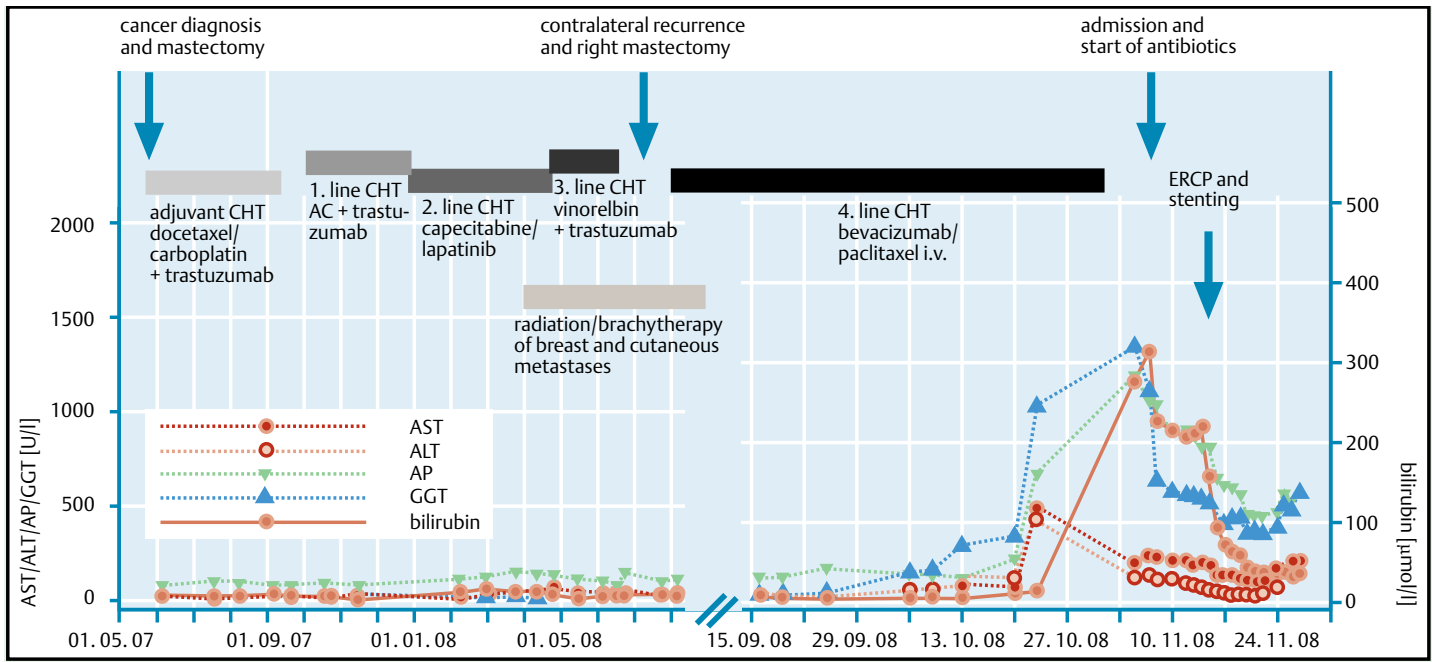

Antibiotic therapy and endoscopic retrograde cholangioscopy (ERCP) led to a substantial decrease in cholestatic paramdase/bilirubin) and aspartate aminotransferase (AST)/alanine eters (alkaline phosphatase (AP)/gammaglutamyltranspepti-

aminotransferase (ALT). CHT, chemotherapy; AC, cyclophosphamide/doxorubicin.
A 59-year-old woman was referred to our hospital by her gynaecologist because of progressive jaundice. She had been diagnosed as having left-sided breast cancer 18 months ago. Two months before admission, she had started chemotherapy with intravenous paclitaxel $\left(90 \mathrm{mg} / \mathrm{m}^{2}\right)$ and bevacizumab (10 mg/kg). Fig. 1 summarizes the patient's treatments and results of the liver function tests (LFTs).

On admission, blood tests revealed markedly elevated LFTs in a cholestatic pattern and raised levels of C-reactive protein. There was no evidence of underlying viral, metabolic, or autoimmune liver disease, and antibiotic therapy was initiated. A contrast-enhanced abdominal ultrasound and magnetic resonance (MR) scan revealed remarkably normal liver parenchyma ( Fig. 2) but moderate intrahepatic cholestasis. Subsequently, endoscopic retrograde cholangioscopy (ERCP) was performed, which showed a completely stenosed, but negotiable, common bile duct (CBD) with prestenotic intrahepatic duct dilatation ( $\bullet$ Fig. 3). On the basis of the above findings, secondary sclerosing cholangitis (SSC) was diagnosed. Following the antibiotic therapy and endoscopic intervention, there was a substantial decrease in cholestasis (๑ Fig. 1).
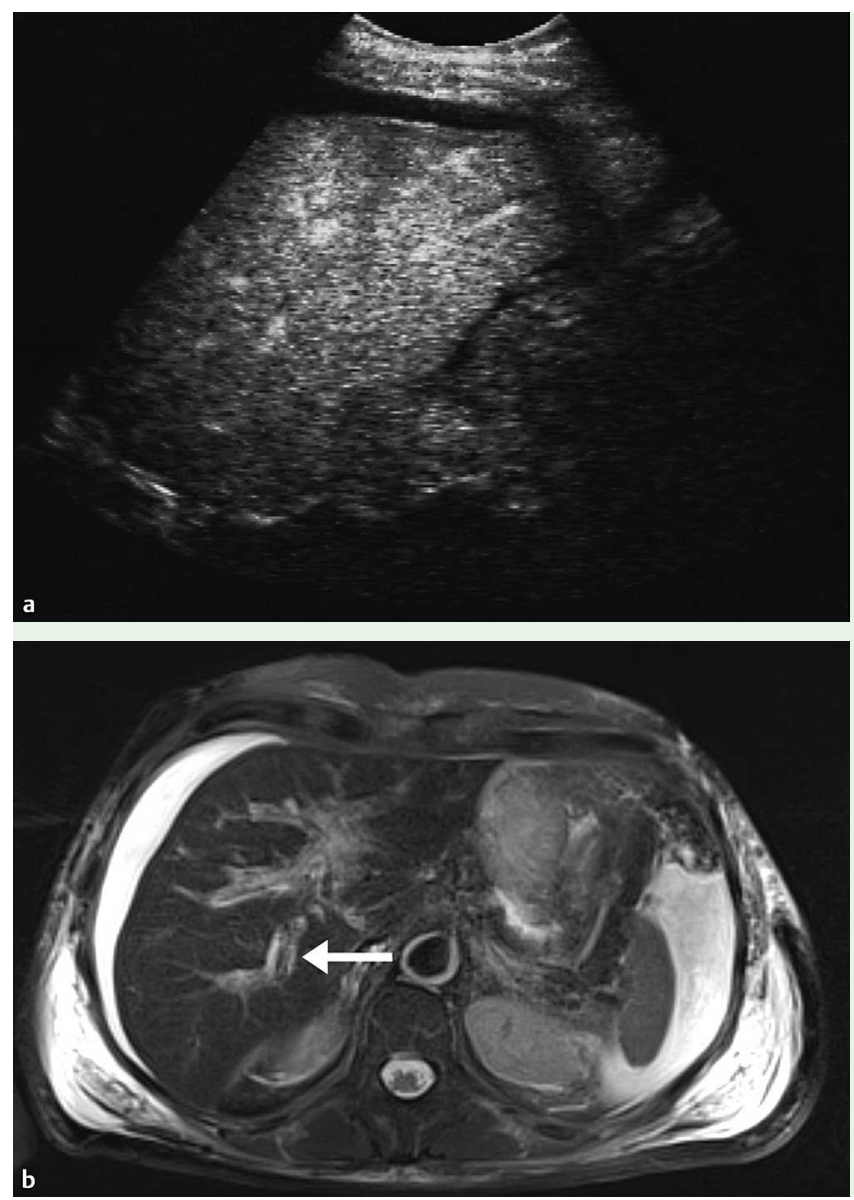

Fig. 1 Treatments received and liver function test (LFT) results before and after the patient's admission. The previous chemotherapy treatments had not led to any changes in the LFTs. However, following initiation of chemotherapy consisting of bevacizumab and paclitaxel there was a dramatic increase in the LFTs.

Fig. 2 Diagnostic imaging of liver. a Contrast-enhanced ultrasound study of the liver showing normal appearing parenchyma with no signs of malignant infiltration of the liver. b T2-weighted magnetic resonance scan confirmed normal liver parenchyma with no evidence of compression of the common bile duct by intraabdominal tumor. Moderate, centrally localized intrahepatic cholestasis was apparent (arrow). Note the perihepatic ascites. 

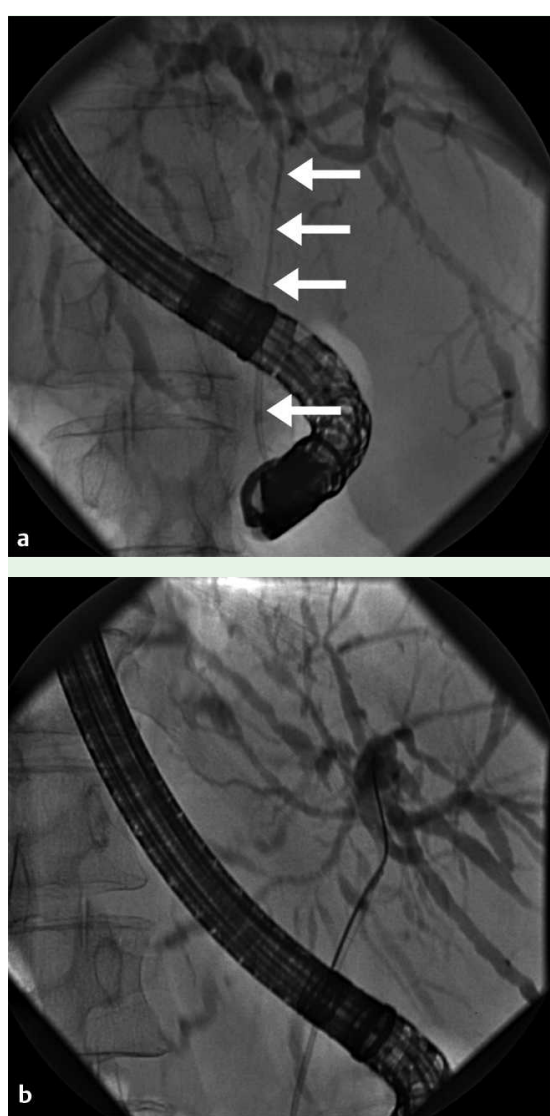

Fig. 3 Endoscopic retrograde cholangioscopy. a Nearly complete stenosis of the common bile duct (arrow), including the hilar region. The intrahepatic ducts are prestenotically dilated with no signs of strictures. b Negotiating the common bile duct stenosis with a wire.
SSC is a rare disease, characterized by bile duct inflammation, fibrosis, and strictures $[1,2]$. It was recently reported that treatment with intravenous bevacizumab caused SSC in a patient with colorectal carcinoma after surgical treatment of liver metastases [3]. In our case, the time course of the disease suggests a link with the chemotherapy with paclitaxel and bevacizumab. Bevacizumab seemed more likely to be the causal agent, because the patient had previously well tolerated a synthetic analog of paclitaxel. Ischemic cholangitis - a known cause of SSC [4] may be induced by the prothrombotic antibody bevacizumab by clot formation in the arterioles supplying the bile ducts. This may explain why in our patient the CBD and not the intrahepatic bile ducts was selectively affected, since both compartments are supplied by distinct vessels. The development of SSC in a previously normal liver underlines the importance of being aware of the hepatic adverse effects of bevacizumab.

Endoscopy_UCTN_Code_CCL_1AZ_2AJ

G. von Figura ${ }^{1}$, J. Stephani ${ }^{1}$, M. Wagner ${ }^{1}$, M. Wegener ${ }^{2}$, C. Wolf $^{3}$, G. Adler ${ }^{1}$,

\section{H. Kulaksiz ${ }^{1}$}

1 Department of Internal Medicine I, University of Ulm, Germany

2 Department of Radiology, University of Ulm, Germany

3 Department of Gynaecology, Medical Center Ulm, Germany

\section{References}

1 Abdalian R, Heathcote EJ. Sclerosing cholangitis: a focus on secondary causes. Hepatology 2006; 44: 1063 - 1074

2 Kulaksiz H, Heuberger D, Engler $S$ et al. Poor outcome in progressive sclerosing cholangitis after septic shock. Endoscopy 2008; 40: 214-218

3 Delis S, Triantopoulou C, Bakoyiannis A et al. Sclerosing cholangitis in the era of target chemotherapy: a possible anti-VEGF effect. Dig Liver Dis 2009; 41: 72 - 77

4 Batts KP. Ischemic cholangitis. Mayo Clin Proc 1998; 73: 380-385

\section{Bibliography}

DOI $10.1055 / \mathrm{s}-0029-1214705$

Endoscopy 2009; 41: E153-E154

(c) Georg Thieme Verlag KG Stuttgart · New York . ISSN 0013-726X

\section{Corresponding author}

\section{H. Kulaksiz, MD}

Department of Internal Medicine I

University of Ulm

Albert-Einstein-Allee 23

$89081 \mathrm{Ulm}$

Germany

Fax: +49-731-50045226

hasan.kulaksiz@uniklinik-ulm.de 